\title{
Alpha methyl acyl CoA racemase deficiency: Diagnosis with isolated elevated liver enzymes
}

\author{
Mehmet Gündüz ${ }^{1}$, Özlem Ünal ${ }^{1}$, Aynur Küçükçongar-Yavaş ${ }^{1}$, Çiğgem Kasapkara ${ }^{2}$ \\ ${ }^{1}$ Division of Metabolism and Nutrition, Ankara Child Health and Diseases, Hematology Oncology Training and Research \\ Hospital, University of Health Science, Ankara; ${ }^{2}$ Department of Pediatric Metabolism and Nutrition, Dr. Sami Ulus \\ Maternity and Children's Training and Research Hospital, University of Health Sciences, Ankara, Turkey. \\ E-mail: unalozlem@gmail.com
}

Received: 22nd January 2018, Revised: 24th March 2018, Accepted: 31st March 2018

SUMMARY: Gündüz M, Ünal Ö, Küçükçongar-Yavaş A, Kasapkara Ç. Alpha methyl acyl CoA racemase deficiency: Diagnosis with isolated elevated liver enzymes. Turk J Pediatr 2019; 61: 289-291.

\begin{abstract}
Alpha methy acyl CoA racemase (AMACR) deficiency is a rare autosomal recessive peroxisomal disorder characterized by cholestatic liver disease in the neonatal period, and variable neurologic symptoms affecting central and peripheral nervous systems in the following years. We report a Turkish patient who was diagnosed with AMACR deficiency with presentation of isolated elevated liver enzymes. The patient was referred for elevated liver enzymes when he was 10 months old. He had no cholestasis history in the neonatal period. Initially, an etiology could not be identified. Ultimately, the patient was diagnosed with AMACR deficiency with previously unreported p.Cys20Tyr (c.596G>A) homozygous pathogenic variant. At last visit, when he was 7.5 years old, his growth, development and neurologic examination were all normal. Biochemical analysis was normal except for mildly elevated AST levels. We suggest that checking VLCFA analysis may be useful in isolated elevated liver enzymes with unknown etiology.
\end{abstract}

Key words: alpha methyl acyl-CoA racemase (AMACR) deficiency, peroxisomal disorders, bile acids, elevated liver enzymes.

Alpha methyl acyl-CoA racemase (AMACR) deficiency is a rare autosomal recessive disorder caused by a deficiency of the AMACR enzyme which is found in peroxisomes and the mitochondria. It is caused by mutations in AMACR gene on chromosome 5p13.2-5q11.1. AMACR is responsible in the lipid metabolism from the elimination of methyl-branched fatty acids and synthesis of bile acids. Serum pristanic acid and C27 bile acid intermediates are increased in AMACR deficiency.

The disorder is characterized by cholestatic liver disease in the neonatal period, and variable neurodegenerative symptoms affecting both central and peripheral nervous systems in the following years. ${ }^{1-6}$ It has a variable age of onset from newborn period to late adulthood. Clinical features may include rhabdomyolysis ${ }^{7}$, seizures, visual failure, spasticity, migraine, ataxia, dysarthria, sensorimotor neuropathy, depression, and white matter hyperintensities in various brain regions on brain imaging.

Herein we report a Turkish patient diagnosed as AMACR deficiency who presented with isolated elevated liver enzymes.

\section{Case Report}

A 10-month-old boy was referred to Pediatric Metabolic Unit with elevated liver enzymes. He was born at term with a birth weight of 3,500 g. He was the sixth child of consanguineous Turkish parents. Parents were first degree cousins. There was no family history of metabolic diseases. He had been followed up from the newborn period with serial high liver enzymes at another hospital. He had no cholestasis history in neonatal period. On admission, he had mild hepatosplenomegaly. Laboratory tests showed the following findings: hemoglobin $12.6 \mathrm{~g} / \mathrm{dl}$, white blood cell count $18,100 / \mathrm{mm}^{3}$, platelet $382,000 /$ 
$\mathrm{mm}^{3}$, alanine aminotransferase (ALT) 376 $\mathrm{U} / \mathrm{L}(\mathrm{N}: 5-45)$, aspartate aminotransferase AST 199 U/L (N: 20-60), gama glutamyl transferase (GGT) $31 \mathrm{U} / \mathrm{L}(\mathrm{N}: 0-40)$, total protein $5.4 \mathrm{~g} / \mathrm{dl}$, albumin $3.9 \mathrm{~g} / \mathrm{dl}$, alkaline phosphatase (ALP) $165 \mathrm{U} / \mathrm{L}(\mathrm{N}: 91-375)$, total bilirubin $1.3 \mathrm{mg} / \mathrm{dl}$, conjugated bilirubin 0.5 $\mathrm{mg} / \mathrm{dl}$, INR 0.97 (0.86-1.2), activated partial thromboplastin time 28.2 seconds (N: 27.938.1). Abdominal ultrasonography showed normal liver parenchyma echogenicity. Serum lipid profile, alpha-1 antitrypsin level, plasma and urine amino acid analysis, tandem mass spectroscopy and urine organic acid analysis, ophtalmologic examination, were all normal.

At follow up visit, when he was 2 years old, his growth parameters were normal, and liver was palpable $2 \mathrm{~cm}$ below costal margin. Laboratory investigations revealed: ALT: $38 \mathrm{U} / \mathrm{L}(\mathrm{N}: 5-45)$, AST 67 U/L (N: 20-60), GGT 8 U/L (N: 0-40), ALP $577 \mathrm{U} / \mathrm{L}$ (N: 91-375). Mild heterogeneity and granular pattern in the liver was found on abdominal ultrasonography.

Very long chain fatty acid (VLCFA) analysis showed elevated pristanic acid $(28.34 \mu \mathrm{mol} / \mathrm{L}$ $\mathrm{N}: 0-1.5)$ and phytanic acid $(4.37 \mu \mathrm{mol} / \mathrm{L} ; \mathrm{N}$ : 0.42-3.77) levels. C26:0, C24:0, C26:0/C22:0 ratio were normal. Cranial magnetic resonance imaging (MRI) revealed terminal zones of myelination area located at the posterior aspect of the lateral ventricles exhibiting at high T2 signal.

Next Generation Sequencing Technique was used for mutation screening in AMACR gene by Illumina-Miseq (Illumina, San Diego, CA) by using in-house designed primers. The Integrative Genomics Viewer (IGV) software of Broad Institute was used for analysis and comparison with reference sequence. AMACR deficiency was suggested with VLCFA analysis and, a previously unreported (novel) NM_001167595.1:c.59G >A (p.Cys20Tyr) homozygous pathogenic variant was found in the AMACR gene. The variant was not present HGMD-Public version, ClinVar, 1000 genome database, EXAC and 2000 exome data of Intergen Genetics Center and also in Google search. It was evaluated with ACMG criteria and evaluated as Variant of Uncertain Significance but in silico analysis several computational programs showed, 8 pathogenic predictions from DANN, GERP, LRT,
MetaSVM, MutationAssessor, MutationTaster, Polyphen 2 and PROVEAN vs 2 benign predictions from dbNSFP.FATHMM and MetaLR. Parents were heterozygotes. VLCFA analysis and liver enzymes were normal in the parents and siblings.

At last visit, when he was 7.5 years old, his growth parameters and neurologic examination were normal. Biochemical analysis was normal except for mildly elevated AST level. Liver enzymes were as follows: AST $69 \mathrm{U} / \mathrm{L}(0-50)$, ALT $44 \mathrm{U} / \mathrm{L}$ (0-50), GGT $13 \mathrm{U} / \mathrm{L}$ (3-22), ALP $232 \mathrm{U} / \mathrm{L}$ (86-315). Coagulation parameters were normal. Abdominal ultrasonography was found to be completely normal. Ophtalmologic examination, hearing assessment were normal. Cranial MRI was reevaluated and it was normal. Informed consent was received from the family for publication of the clinical information.

\section{Discussion}

Neurological manifestations in AMACR deficiency including developmental delay, epilepsy, acute encephalopathy, tremor, pigmentary retinopathy, hemiparesis, spastic paraparesis, peripheral neuropathy, depression, headache, cognitive decline and ataxia can start at any age from childhood to late adult life. Analysis of plasma bile acids show increased concentrations of DHCA and THCA. Plasma fatty acid analysis shows an elevated concentration of pristanic acid with mildly elevated or normal phytanic acid concentration and normal very long chain fatty acids. ${ }^{8}$

Clinical findings are highly variable in AMACR deficiency. Ferdinandusse et al. ${ }^{1}$ described three patients with elevated plasma concentrations of pristanic acid and C27-bile-acid intermediates. Two of those patients suffered from adult-onset sensory motor neuropathy. One patient also had pigmentary retinopathy, suggesting Refsum disease, whereas the other patient had upper motor neuron signs in the legs, suggesting adrenomyeloneuropathy. The third patient was a child without neuropathy. Smith et al. ${ }^{9}$ described a 45-year-old male with a history of seizures who presented with relapsing encephalopathy. AMACR deficiency was also reported to be associated with acute rhabdomyolysis. ${ }^{7}$ Our patient was diagnosed with AMACR with very subtle findings including mildly elevated liver enzymes and mild hepatosplenomegaly 
in early childhood. He had no neonatal cholestasis, neurologic, sensorial findings and both cranial and abdominal imagings were normal. Diagnosis with only elevated liver enzymes without cholestasis in the childhood is a highly unusual presentation for AMACR deficiency.

Pathogenic variants in adults with neurological disease included a common mutation, p.Ser52Pro, and p.Leu107Pro. ${ }^{1,4,5,6}$ P.Ser52Pro pathogenic variant was also reported in patients presenting as neonatal cholestasis. ${ }^{3}$ p.Cys20Tyr (c.596G >A) homozygous pathogenic variant was found in our patient, and it is not a predictive common mutation for neurological disease in the adult period.

Characteristic degeneration of cerebellar afferents and efferents, including the dentatothalamic tract and thalamic lesions on magnetic resonance imaging studies may assist in diagnosis. ${ }^{10}$ Cranial MRI was normal and there were no neurologic findings at 7.5 years of age in our patient but it is important to follow up this patient in terms of neurologic findings and white matter pathologies.

We discussed a child with unusual presentation of AMACR deficiency. We suggest that checking VLCFA analysis may be useful in isolated elevated liver enzymes with unknown etiology.

\section{REFERENCES}

1. Ferdinandusse S, Denis S, Clayton PT, et al. Mutations in the gene encoding peroxisomal alpha-methylacyl-CoA racemase cause adult-onset sensory motor neuropathy. Nature Genet 2000; 24: 188-191.
2. Van Veldhoven PP, Meyhi E, Squires RH, et al Fibroblast studies documenting a case of peroxisomal 2-methylacyl-CoA racemase deficiency: possible link between racemase deficiency and malabsorption and vitamin $\mathrm{K}$ deficiency. Eur J Clin Invest 2001; 31: 714-722.

3. Setchell KD, Heubi JE, Bove KE, et al. Liver disease caused by failure to racemize trihydroxycholestanoic acid: gene mutation and effect of bile acid therapy. Gastroenterology 2003; 124: 217-232.

4. Clarke CE, Alger S, Preece MA, et al. Tremor and deep white matter changes in alpha-methylacyl-CoA racemase deficiency. Neurology 2004; 63: 188-189.

5. Thompson SA, Calvin J, Hogg S, Ferdinandusse S, Wanders RJA, Barker RA. Relapsing encephalopathy in a patient with alpha-methylacyl-CoA racemase deficiency. J Neurol Neurosurg Psychiatry 2008; 79: 448-450.

6. Dick D, Horvath R, Chinnery PF. AMACR mutations cause late-onset autosomal recessive cerebellar ataxia. Neurology 2011; 76: 1768-1770.

7. Hamel Y, Mamoune A, Mauvais FX, et al. Acute rhabdomyolysis and inflammation. J Inherit Metab Dis 2015; 38: 621-628.

8. Wanders RJA, Aubourg P, Poll-The BT. Inborn errors of non-mitochondrial fatty acid metabolism including peroxisomal disorders. In: Saudubray JM, Baumgartner MR, Walter JH (ed). Inborn Metabolic Diseases (6 ${ }^{\text {th }}$ ed). Berlin, Heidelberg: Springer, 2016: 597-598.

9. Smith EH, Gavrilov DK, Oglesbee D, et al. An adult onset case of alpha-methyl-acyl-CoA racemase deficiency. J Inherit Metab Dis 2010; 33(Suppl 3): S349-S353.

10. Haugarvoll K, Johansson S, Tzoulis C, et al. MRI characterisation of adult onset alpha-methylacyl-coA racemase deficiency diagnosed by exome sequencing. Orphanet J Rare Dis 2013; 8: 1. 